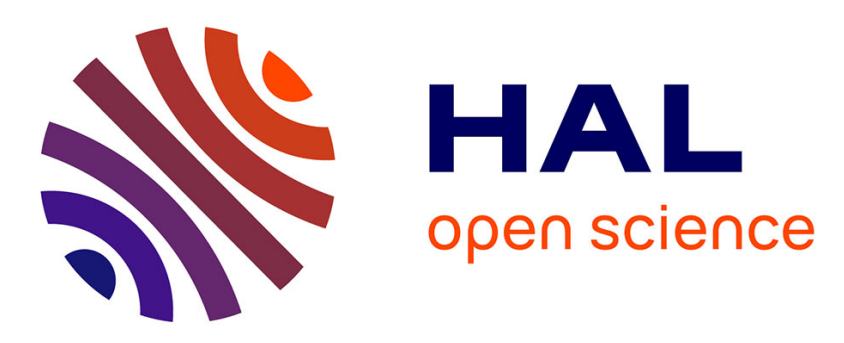

\title{
Direct injection in organic SU8 nanowires and nanotubes for waveguiding properties investigation
}

John Bigeon, Nolwenn Huby, Jean-Luc Duvail, Bruno Bêche

\section{To cite this version:}

John Bigeon, Nolwenn Huby, Jean-Luc Duvail, Bruno Bêche. Direct injection in organic SU8 nanowires and nanotubes for waveguiding properties investigation. Proceedings of SPIE, the International Society for Optical Engineering, 2014, Proc. SPIE 9126, Nanophotonics V, pp.6. $10.1117 / 12.2052517$. hal-00981482

\section{HAL Id: hal-00981482 \\ https://hal.science/hal-00981482}

Submitted on 22 Apr 2014

HAL is a multi-disciplinary open access archive for the deposit and dissemination of scientific research documents, whether they are published or not. The documents may come from teaching and research institutions in France or abroad, or from public or private research centers.
L'archive ouverte pluridisciplinaire HAL, est destinée au dépôt et à la diffusion de documents scientifiques de niveau recherche, publiés ou non, émanant des établissements d'enseignement et de recherche français ou étrangers, des laboratoires publics ou privés. 


\title{
Direct injection in organic SU8 nanowires and nanotubes for waveguiding properties investigation
}

\author{
Bigeon J. ${ }^{a}$, Huby N. ${ }^{a}$, Duvail J-L ${ }^{b}$ and Bêche B. ${ }^{a, c}$ \\ ${ }^{a}$ Institut de Physique de Rennes, Université de Rennes 1, CNRS UMR 6251, Rennes, France \\ ${ }^{b}$ Institut des Matériaux de Nantes Jean Rouxel, Université de Nantes, CNRS UMR 6502, \\ Nantes, France \\ ${ }^{c}$ Institut universitaire de France, IUF, Paris, France
}

\begin{abstract}
We report photonic concepts related to injection and sub-wavelength propagation in nanofibers (nanowires and nanotubes). These nanostructures are fabricated by the wetting template method leading to aspect ratio of over 250. At first, injection into nanowires and nanotubes of SU8, a photoresist used for integrated photonics, was successfully achieved by using polymer microlensed fibers with sub-micronic radius of curvature. Theoretical simulation by finite domain time-dependent (FDTD) method was used to determine the sub-wavelength propagation for nanowires and nanotubes and corroborate this coupling phenomena. The original confinment of energy density into SU8 nanotubes is highlighted. Finally, characterisation of propagation losses is reported by using a cut-back method transposed to such nanotubes and determined to range between 1 and $2 \mathrm{~dB} / \mathrm{mm}$. Both injection and cut-back method developed here are compatible with any sub-micronic structures. This work on SU8 nanofibers suggests broader perspectives for future nanophotonics.
\end{abstract}

Keywords: nanophotonics, sub-wavelength propagation, SU8, polymer, wetting-template, nanotube, nanowire, cut-back method

\section{INTRODUCTION}

Sub-wavelength waveguides are the subject of numerous publications and applications ${ }^{1}$ (Mach-Zehnder, add-drop filter, etc...). Due to their sub-wavelength dimensions, 1D-nanostructures are of particular interest in integrated optics by the ability to control their aspect ratio. Effective light waveguiding has been well investigated mainly in silica nanofibers while only few studies have been reported in organic nanofibers. The SU8 polymer was selected, because it is a widely-used epoxy based negative photoresist, and commonly used in integrated photonics, due to its excellent characteristics for micronic waveguides. ${ }^{2}$ SU8 polymer presents large refractive index compared to most organics and polymers. This large refractive index enhances the confinment in light waveguiding. Further, the tubular aspect of nanotube give a new degree of modulation in photonics. In literature, Zhao et al. ${ }^{3}$ have shown that the tubular geometry of nanotube can uniquely confine part of the energy density in the lowest refractive index area, the core of the nanotube. This original confinment allows tuning of light propagation and light-matter interaction. In this present work, we detail keys for: low-cost production of SU8 polymer nanowires and nanotubes by wetting template method, experimental protocol to directly inject light into such nanostructures, numerical analysis of sub-wavelength propagation, and finally characterisation of optical losses for SU8 nanotubes.

\section{SYNTHESIS}

The wetting template method allows the creation of 1D nanostructures with high aspect ratio, diameter ranging from $50 \mathrm{~nm}$ to $250 \mathrm{~nm}$ and length up to $100 \mu \mathrm{m}$ typically. This method achieves low-cost fabrication, fine control dimensions while providing the possibility of large-scale production. This method is based on the use of a previously nanostructured template, pores with nanometer dimensions, which allows to organise the growth

Further author information: (Send correspondence to Huby N.)

Huby N.: E-mail: nolwenn.huby@univ-rennes1.fr, Telephone: +33 (0)2 23236225 
of nanomaterials. This link between morphology and nanomaterial template allows control of size, shape and orientation of the nanofibers. In our study, AAO (Anodic Aluminium Oxide) membrane presents perfectly cylindrical pores arranged in the plane perpendicular to the axis of the pores, as visible on figure 1a.

Wetting template method ensures wetting of AAO by the material in a liquid phase due to the surface energy of the alumina. Here, we used the SU8 photoresist in solvent. Additionally, the different viscosities of SU8 available in liquid phase are a central advantage for the template strategy. Elaboration of SU8 nanowires and nanotubes is selected by the difference of surface energy between the polymer in liquid phase and the alumina. High or low viscosity of solution leads to synthesis of nanowires or nanotubes respectively.

Once the in-solution polymer penetrates over the entire thickness, a UV insolation $\left(\lambda=365 \mathrm{~nm}, 200 \mathrm{~mJ} / \mathrm{cm}^{2}\right)$ promotes cross-linking of the SU8 to get quite rigid nanofibers. A selective chemical etching by $\mathrm{H}_{3} \mathrm{PO}_{4}$ solution, releases SU8 nanofibers from the AAO. Finally, SU8 nanofibers were dispersed into isopropyl alcohol or distilled water. A drop of the suspension of SU8 nanofibers was deposited onto a clean $\mathrm{SiO}_{2}$ wafer. Figure $1 \mathrm{~b}$ and $1 \mathrm{c}$ displays scanning electron microscopy $(S E M)$ images of SU8 nanotubes and SU8 nanowires respectively after partial release from the AAO membrane. No residual material and a smooth surface confirm fabrication process and purification protocols. SU8 nanofibers present regular and hollow endfacets. External diameter of $240 \mathrm{~nm}$ was extrapolated for nanowires and nanotubes (for an average inner diameter of $120 \mathrm{~nm}$ ).

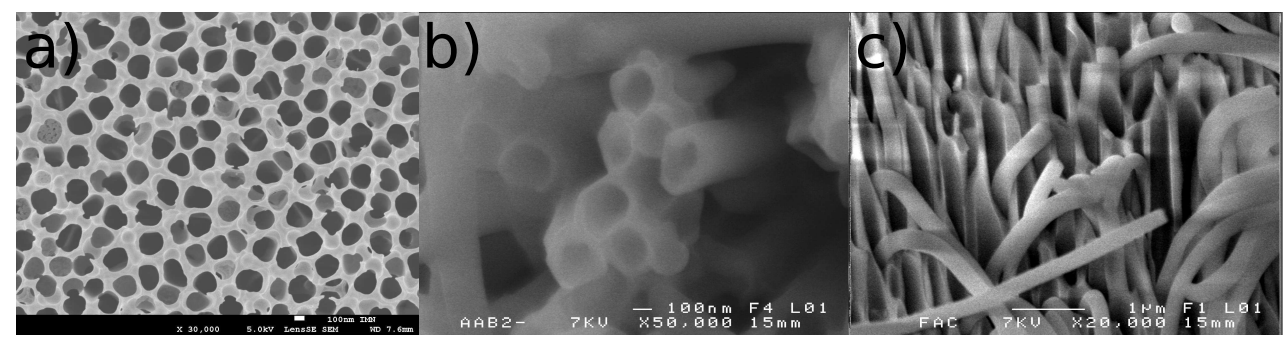

Figure 1. a) SEM top-view image of AAO membrane with cylindrical nanopores. SEM image of b) SU8 nanotubes and c) SU8 nanowires elaborated by the wetting template method after the partial release from the AAO membrane.

\section{EXPERIMENTAL SET-UP}

In literature, the injection in light-guiding sub-micronic structures is based on two different mechanisms : by grating side couplers ${ }^{4}$ or through evanescent coupling between two photonic structures. ${ }^{1}$ However, these approaches require micronic coupling distances and do not allow to fully optimise the quality of the injection in nanostructures nanoscale. In previous publications, ${ }^{5,6}$ we have reported the single-mode behavior hybrid mode $H E_{11}$ of SU8 polymer nanowires and nanotubes by theoretical and experimental approach. Light injection was accomplished by evanescent coupling with SU8 sub-micronic structures. ${ }^{5}$

Here, an innovative way to inject light into 1D nanostructures has been developed by using polymer microlens. The injection of the light is provided by single-mode (@630 nm) microlensed polymer fiber (Lovalite). In addition, microlens fibers are positioned by piezo-electric nano-positionner (Pi-611-SF) in order to precisely control their positioning with $10 \mathrm{~nm}$ step in the three directions and optimise light injection inside nanostructures. A top-view display system, figure 2, consisting of a monochrome CCD camera (AVT GX1920), with pixels size of $4.54 \mu \mathrm{m}$ associated with $\mathrm{x} 40$ and x100 optical lenses (numerical aperture 0.90, working distance $1 \mathrm{~mm}$ ) permits the simultaneous control and optimisation of the light injection in nanostructures. A beam-splitter element on optical path allows the spectral detection of nanostructures by spectrometer (OCEAN Optics USB2000).

\section{SUB-WAVELENGTH PROPAGATION}

Figure 3a displays a SEM image of a microlensed fiber which present at their end a curvature radius of $500 \mathrm{~nm}$. The cylindrical geometry of the microlens has been chosen to coincide with the tubular symmetry of SU8 nanofibers. Thereby, the energy overlap integral between the injecting microlens and the SU8 nanofibers is maximised. Under laser excitation $(675 \mathrm{~nm})$, microlens polymer fibers exhibit a light lobe typically of a few micrometers in diameter. Figure $3 \mathrm{~b}$ presents the approach phase of the microlensed fiber near the SU8 nanotube 


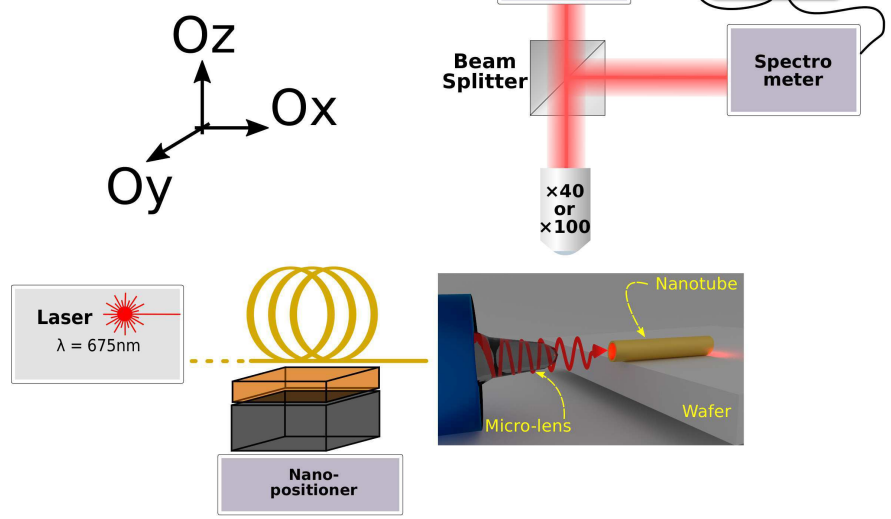

Figure 2. Sketch of top-view display system to control and optimise the direct light injection into 1D nanostructures. A microlensed fiber is mounted on piezo-electric nano-positionner. CCD Camera or spectrometer allow optical analysis of coupling phenomena.

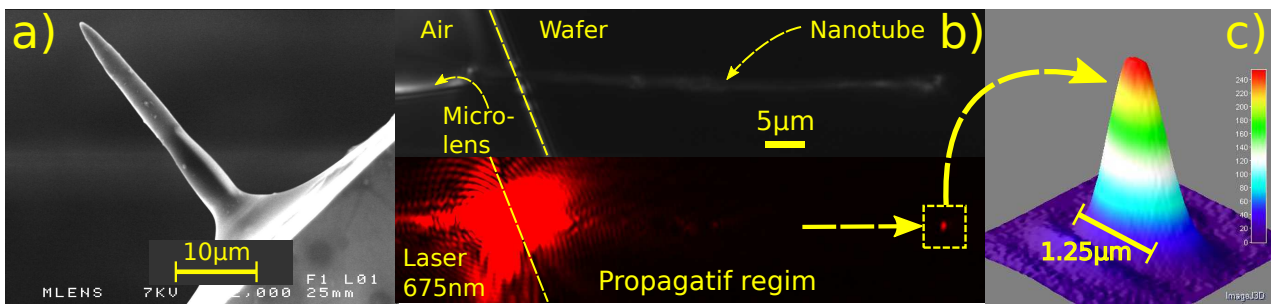

Figure 3. a) SEM image of the microlensed polymer fiber for direct injection into nanotube. b) Top-view image of the approach phase of the microlensed fiber near the SU8 nanotube under visible light and under laser excitation (675 nm). c) 3D-view of output propagative spot at the end of the SU8 nanotube.

under visible light. After an adequate protocol of positioning optimised by the top-view display system, the microlens is brought thanks to the piezo control at few micrometers of the nanotube. On the figure $3 \mathrm{~b}$, when the microlens is located close to the SU8 nanotube, under laser excitation at $675 \mathrm{~nm}$, a spot at the opposite end of the nanotube is visible. The presence of this spot, highlighted by $3 \mathrm{D}$-view on figure 3c, is the proof of a significant injection into the SU8 nanotube. The absence of light scattering along the organic SU8 nanotube is an indicator of low roughness of the surface of the nanotube and the absence of morphological defects. An identical behavior has been determined for SU8 nanowires.

\section{FDTD MODELISATION}

The sub-wavelength propagation of nanowires and nanotubes has been investigated by a numerical 3D finite domain time-dependent (FDTD) simulation and performed by Meep freeware. ${ }^{7}$ FDTD simulation has already proved accuracy to study optical sub-wavelength propagation in nanostructures. ${ }^{8,9}$ As determined by figure 1b,c in section 2, geometrical parameters of SU8 nanowires are set as an external diameter of $240 \mathrm{~nm}$ for SU8 nanowires and nanotubes, and an inner diameter of $120 \mathrm{~nm}$ for nanotubes. The model contains an Oy-polarised laser source at $675 \mathrm{~nm}(\mathrm{FWHM}=12 \mathrm{~nm})$ launched inside nanowires and nanotubes with refractive index of $n_{S U 8}=1.56$.

Figures $4 \mathrm{a}$ and $4 \mathrm{~b}$ present orthogonal views (i.e. : along Oyz plan) of the optical power mode $H E_{11}$ inside the SU8 nanowires and nanotubes. It highlights the separate behavior of density energy confinment of both nanostructures. In case of nanowires, the maximum of light is confined into the SU8 nanofibers. In case of nanotubes, the tubular geometry allows a significant confinment of density power inside the hollow region, the 
maximum of light propagating in the wall of the nanotube with a extension of the density power of $50 \mathrm{~nm}$ in air. This makes nanotubes based devices sensitive to surface modification and offers great opportunities for near-field sensors.

In such sub-wavelength propagation regime, the optical coupling between the microlensed fiber and the SU8 nanofibers is critical and deserves a specific study. Figure 4c is a top-view of the power density of the microlensed fiber aligned in direction of free-standing SU8 nanotube. The presence of standing wave patterns along the axis of nanotube attest the coupling phenomena between SU8 nanotube and microlensed fibers. They are due to interference between propagating light and reflection at the output endfacet. This phenomena has been already established on waveguiding silica nanowires. ${ }^{10}$ Projection of power density inside the nanotube along Ox on figure 4 a corroborates the period of standing waves : $P=\frac{\lambda}{2 n_{e f f}} \cong 332 \mathrm{~nm}$ from our previous theoretical work. ${ }^{6}$
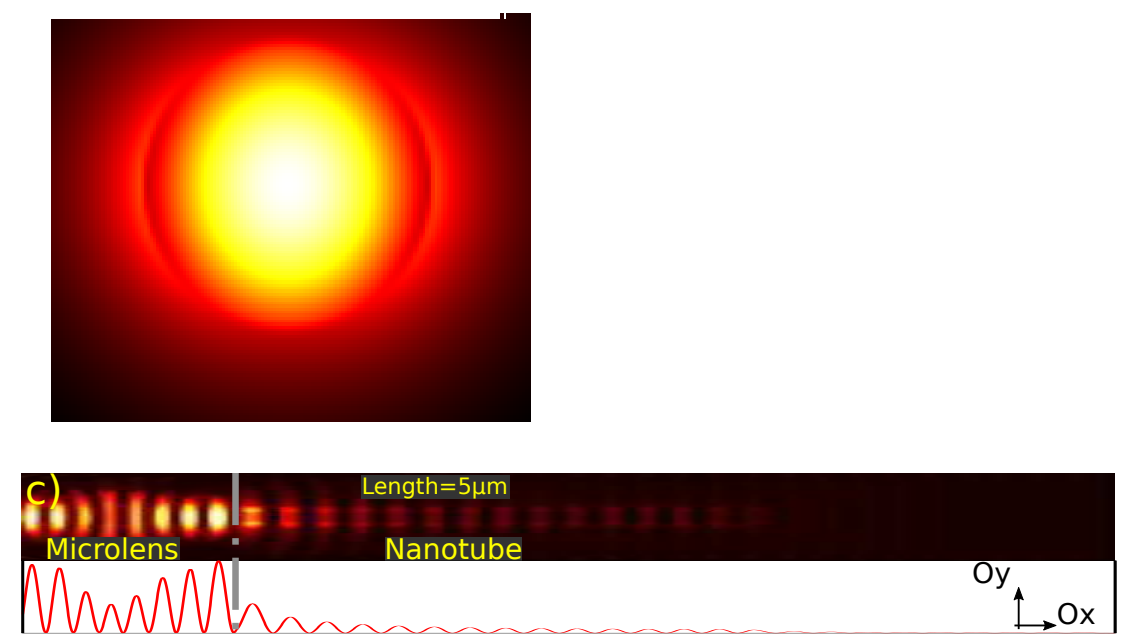


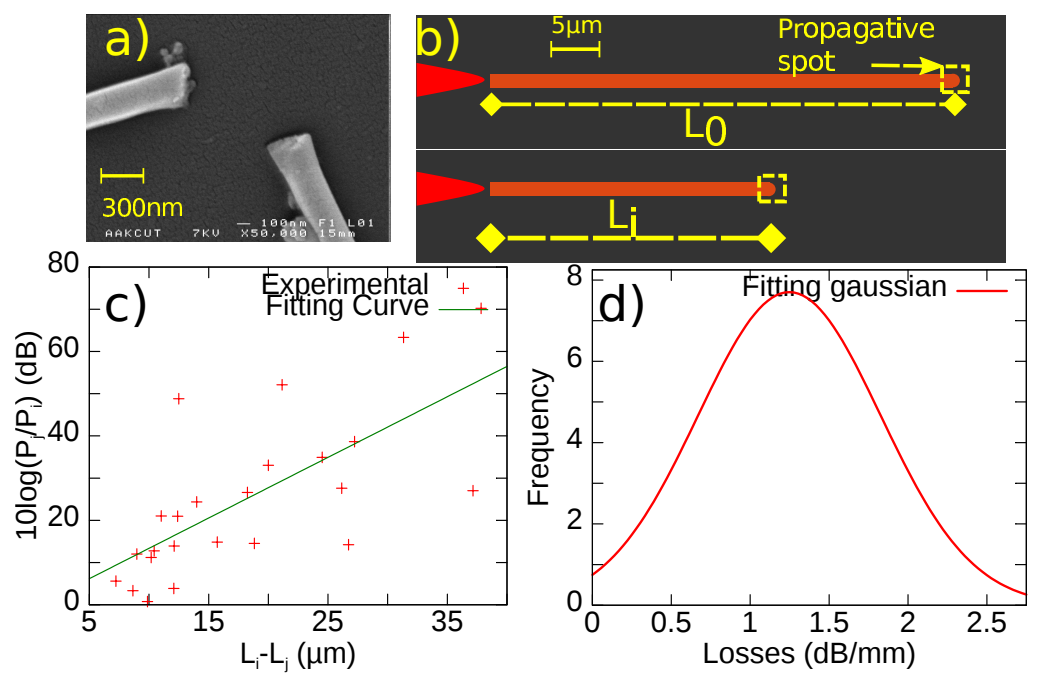

Figure 5. a) SEM image of a clean cleaved endface of SU8 nanotube, b) top-views of images during waveguiding of laser light at different lengths. c) Intensity of propagative spot for different propagation distances and d) distribution of losses determined to range between 1 and $2 \mathrm{~dB} / \mathrm{mm}$.

dispersion of attenuation coefficient summarised on figure $5 \mathrm{~d}$, determined to range between 1 and $2 \mathrm{~dB} / \mathrm{mm}$. For comparison, SU8 microstructures exhibit losses of $17 \mathrm{~dB} / \mathrm{mm} .{ }^{11}$ It can be noted that plasmon nanowaveguides much investigated for sub-wavelength photonics present losses of $4000 \mathrm{~dB} / \mathrm{mm} .{ }^{12}$ In previous work, ${ }^{5}$ scattering losses of SU8 nanotubes had been estimated around $150 \mathrm{~dB} / \mathrm{mm}$. This higher value was allocated to the measurement process (i.e. : scattering), to a synthesis process not optimised, and to a different injection process of light inside SU8 nanotubes (evanescent coupling with microstructures).

\section{CONCLUSION}

In summary, direct injection into organic nanofibers (nanowires and nanotubes) has been demonstrated and confirmed their efficient waveguiding properties. A derivated cut-back method is proposed for attenuation coefficient estimation in such sub-micronic structures. In SU8 nanotubes, the attenuation coefficient is determined to range between 1 and $2 \mathrm{~dB} / \mathrm{mm}$. The sub-wavelength regime propagation in such SU8 nanotubes highlight the modulation of guiding light provided by tubular geometries in nanostructures. This study allows perspectives for SU8 nanotubes as building blocks in more complex systems or sub-wavelength optical probes for near-field interactions. This feature makes polymer SU8 nanotubes a good candidate as the basic element of highly integrated nanophotonics and sub-wavelength optical probes.

\section{ACKNOWLEDGMENTS}

The authors thank Jo Le Lannic and Francis Gouttefangeas for SEM characterization, Guillaume Raffy for FDTD study, Ludovic Frein and Steve Bouhier for helpful discussions and experimental assistance. J. Bigeon $\mathrm{PhD}$ is supported by the Lab-O-Mat french network between CNRS, Université de Nantes and Université de Rennes 1, and Bretagne and Pays de la Loire Regions.

\section{REFERENCES}

[1] Tong, L., Zi, F., Guo, X., and Lou, J., "Optical microfibers and nanofibers: A tutorial," Optics Communications 285, 4641-4647 (Oct. 2012).

[2] Bêche, B., Pelletier, N., Gaviot, E., Hierle, R., Goullet, A., Landesman, J. P., and Zyss, J., "Conception of optical integrated circuits on polymers," Microelectronics Journal 37, 421-427 (May 2006).

[3] Zhao, C., Tang, Z., Ye, Y., Fan, D., Qian, L., Wen, S., and Chen, G., "Field and dispersion properties of subwavelength-diameter hollow optical fiber," Optics Express 15, 6629-6634 (May 2007). 
[4] Saleh, B. E. A. and Teich, M. C., [Fundamentals of Photonics], Wiley-Interscience, 2 ed. (Mar. 2007).

[5] Huby, N., Duvail, J.L., Duval, D., Pluchon, D., and Bêche, B., "Light propagation in single mode polymer nanotubes integrated on photonic circuits," Applied Physics Letters 99, 113302-1-113302-3 (Sept. 2011).

[6] Duval, D. and Bêche, B., "Theoretical formulation to shape versatile propagation characteristics of threelayer-tubular waveguides: sub-wavelength and asymptotic study," Journal of Optics 12, 075501 (July 2010).

[7] Oskooi, A. F., Roundy, D., Ibanescu, M., Bermel, P., Joannopoulos, J., and Johnson, S. G., "Meep: A flexible free-software package for electromagnetic simulations by the FDTD method," Computer Physics Communications 181, 687-702 (Mar. 2010).

[8] Wang, S.-S., Fu, J., Qiu, M., Huang, K.-J., Ma, Z., and Tong, L.-M., "Modeling endface output patterns of optical micro/nanofibers," Optics Express 16, 8887-8895 (June 2008).

[9] Wang, S., Hu, Z., Yu, H., Fang, W., Qiu, M., and Tong, L., "Endface reflectivities of optical nanowires," Optics Express 17, 10881-10886 (June 2009).

[10] Coillet, A., Cluzel, B., Vienne, G., Grelu, P., and Fornel, F. de., "Near-field characterization of glass microfibers on a low-index substrate," Applied Physics B 101, 291-295 (Oct. 2010).

[11] Bêche, B., Pelletier, N., Gaviot, E., and Zyss, J., "Single-mode TE00TM00 optical waveguides on SU-8 polymer," Optics Communications 230, 91-94 (Jan. 2004).

[12] Wang, W., Yang, Q., Fan, F., Xu, H., and Wang, Z. L., "Light propagation in curved silver nanowire plasmonic waveguides," Nano Letters 11, 1603-1608 (Apr. 2011). 